\title{
Cinq ouvrages récents de la voie buissonnière écoformatrice
}

\section{Gaston Pineau}

\section{(2) OpenEdition}

\section{Journals}

Édition électronique

URL : https://journals.openedition.org/ere/3863

DOI : $10.4000 /$ ere.3863

ISSN : 2561-2271

Éditeur

Centr'ERE

Référence électronique

Gaston Pineau, "Cinq ouvrages récents de la voie buissonnière écoformatrice ", Éducation relative à l'environnement [En ligne], Volume 15 - 1 | 2019, mis en ligne le 18 novembre 2019, consulté le 21 octobre 2021. URL : http://journals.openedition.org/ere/3863 ; DOI : https://doi.org/10.4000/ere.3863

Ce document a été généré automatiquement le 21 octobre 2021.

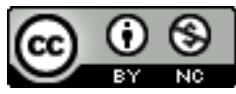

La revue Éducation relative à l'environnement est mise à disposition selon les termes de la Licence Creative Commons Attribution - Pas d'Utilisation Commerciale 4.0 International. 


\section{Cinq ouvrages récents de la voie buissonnière écoformatrice}

\section{Gaston Pineau}

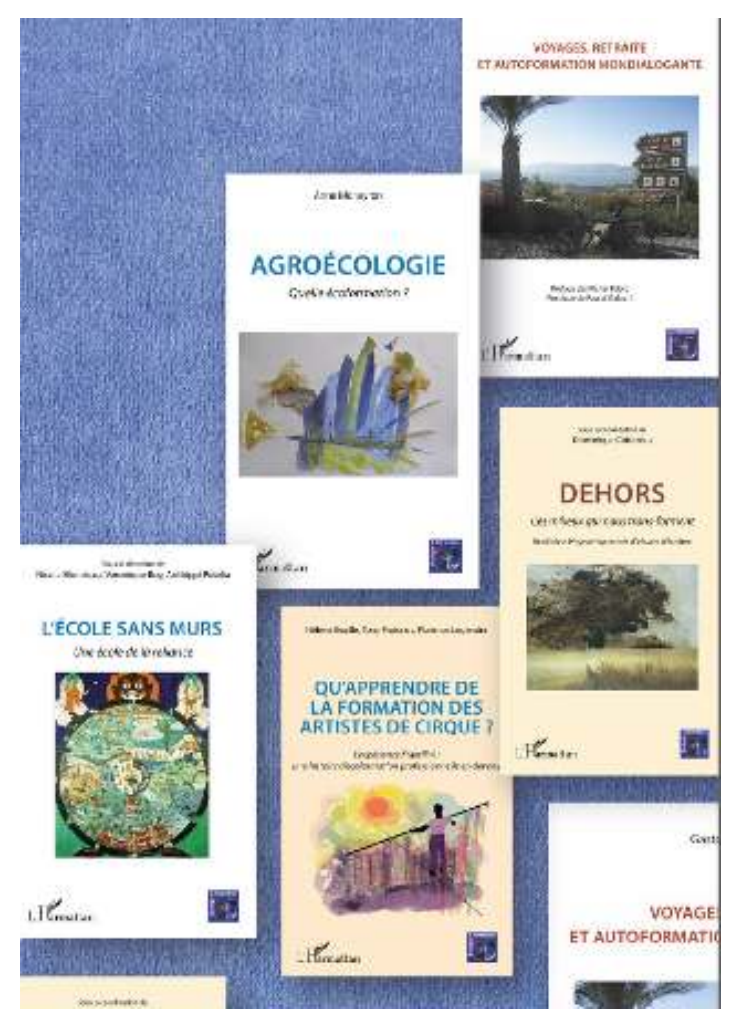

1 Dehors (Cottereau, 2017), en agroécologie (Moneyron, 2018), au cirque (Bézille, Froissart et Legendre, 2018), sans mur (Blondeau, Boy et Potolia, 2019), en dialogue avec le monde (Pineau, 2019), tels sont les lieux de réflexion des cinq derniers ouvrages de la collection Écologie et formation qui empruntent la voie buissonnière très bio-diversifiée de l'écoformation comme démarche de recherche-action-formation avec l'environnement. 
2 C'est en passant en Mongolie avec le Transmongolien en avril 2018 (Pineau, 2018, p. 63) que Véronique Boy m'est revenue en mémoire avec son colloque en 2013 à l'Université de Paris 8 sur L'École sans murs: une autre relation entre les personnes et l'environnement dans l'espace et dans le temps, et sa thèse de 2017, S'éduquer en Bouriatie, anciennement pays nomade. À la recherche des maillons manquants. En 2019, elle revient avec fulgurance avec son ouvrage collectif L'école sans murs. Une école de la reliance (Blondeau, Boy et Potolia, 2019). Elle intitule sa postface : Du colloque à l'ouvrage. Un espace de réflexion.

3 L'esprit d'un colloque peut se diffuser longtemps après qu'il a eu lieu... Revenons sur le questionnement qui a motivé son organisation : ces régions lointaines, sibériennes ou amazoniennes, avec leurs modèles "d'écoles écologiques ", inscrites majoritairement dans des espaces où la nature tient une place encore essentielle, peuvent-elles être de quelque aide à l'école française pour s'ouvrir sur son environnement? Un dialogue peut-il s'instaurer entre les partisans de cet objectif, appartenant à des mondes si différents? (Boy, 2019, p. 249)

Dialogue pas facile - pour y avoir participé - ... en ville, avec des horaires serrés, des amphithéâtres dispersés, des salles pré-programmées, des habitudes pré-conditionnées et des murs pré-fabriqués! Mais l'ouvrage, paru six ans après, atteste qu'il est possible de concevoir et de vivre une école centrée sur la relation à l'environnement. L'École sans mur peut être une école de la reliance. La terre de la réalité (partie 1) peut devenir la réalité des terres (partie 2) et les Espaces métissés, des projets qui nous animent (partie 3).

L'ouvrage coordonné par Dominique Cottereau (2017), Dehors. Ces milieux qui nous transforment. Récits éco-biographiques nés d'ateliers d'écritures, socialise des démarches narratives d'écoformation, orales et écrites en groupe, longuement mûries méthodologiquement par l'auteure. Depuis le début des années 90, et même avant, Dominique Cottereau se forme et nous forment, expérientiellement et formellement : $\grave{A}$ l'école des éléments. Écoformation et classes de mer (Cottereau, 1994). C'est donc une experte pour aider à Tirer le fil écologique de la vie (1 ${ }^{\text {ère }}$ partie), à le tisser et à le tramer avec des moments signifiants ( $2^{\mathrm{e}}$ partie) et Des éléments ( $3^{\mathrm{e}}$ partie) : le hamac, entre ciel et terre; le kayac, pour larguer les amarres, s'évader et se relier; l'eau, entre peur et plaisir à transmettre; la terre et son tonnerre... Autant d'expériences initiatiques qui sont beaucoup plus parlantes et motivantes que les plus beaux cours de didactique !

Il y a des risques, bien sûr. On n'a jamais rien sans rien. Un engagement amène toujours une part de risque. Or comment accompagner l'engagement dans une activité qui implique une prise de risque ? Il faut lire à cet effet Qu'apprendre de la formation des artistes de cirque? (Bézille, Froissart et Legendre, 2018).

À l'ombre du fil tendu du funambule, n'y a-t-il pas ce fil qui relie toute une équipe, celle qui a préparé cette marche aux allures d'impossible ? L'ouvrage se propose de répondre à cette question en passant par l'exploration d'une expérience de formation singulière, ambitieuse, riche d'écoformation professionnelle construite sur la durée : celle du cirque Fratellini et son école attentive au rôle du milieu, du collectif, de la vie quotidienne et des valeurs partagées (solidarité, engagement, émancipation). (4 $4^{e}$ de couverture)

7 Et puis..., vous devez quand même vous nourrir, manger et boire. Ces actes quotidiens dépendent actuellement d'une lutte vitale entre agrobusiness et agroécologie. Alors, vous ne pouvez pas passer à côté de Agroécologie. Quelle écoformation de Anne Moneyron (2018), cette autre pionnière du Groupe de Recherche sur l'Écoformation (GREF) à qui l'on doit: Transhumance et éco-savoir. Reconnaissance des alternances écoformatrices (2003); La méthode Jean Moneyron: une gestuelle thérapeutique de la forme (2006); Temps de vie et 
transhumance. Carnets de voyage d'une Amazone (2012). L'auteure continue son voyage d'Amazone avec un grand "tour d'horizon des agricultures dites alternatives en France" pour passer à l'autrement ( $1^{\text {ère }}$ partie). Elle détaille longuement trois recherchesactions-formations transdisciplinaires: éco-savoirs des Bergers transhumants; écoformation des techniciens d'une Unité Expérimentale (UE) de l'Institut National de la Recherche Agronomique (INRA) ; et une écoformation en commun des viticulteurs de Weshalten. ( $2^{\mathrm{e}}$ partie). Avec la conclusion, Trans-former le Autrement: vers une anthropoformation émancipatrice (p. 143-150), l'auteure termine de façon transcendante - ou plus exactement pour la rejoindre dans son tempo, trans-andante - allant autrement, à son rythme, à travers et au-delà (p. 148) :

Dans la problématique du autrement de l'agroécologie, depuis trente ans, ce qui nous intéresse... ce ne sont pas les états stables d'un avant et d'un après, mais les passages: comment se met-on en actes? Comment se forme l'éco-savoir... la question de leur formation et trans-formation. Il s'agit de considérer, dans la même temporalité de recherche, l'identification et les passages possibles de ces écosavoirs, pour une reconnaissance émancipatrice de leur forme spécifique pour les agricultrices et agriculteurs qui résistent aux modèles imposés, acceptent leur fragilité en acceptant et écoutant leurs intuitions éco-logiques. (p. 149)

Après l'écoformation initiale plus ou moins ouverte à l'environnement socio-naturel (L'école sans murs et Dehors) et des écoformations professionnelles continues (Qu'apprendre de la formation des artistes de cirque et Agroécologie. Quelle écoformation), que reste-t-il pour le troisième âge? Dans la bio-diversité infinie des manières de se former/ transformer avec son milieu habité, à la porte de chacun et en transactions avec les mondes rencontrés, le cinquième ouvrage explore la voie du voyage à la retraite... pour retraiter sa vie: Voyages, retraite et autoformation mondialogante (Pineau, 2019). En 2010, pour sa retraite justement, Christian Verrier a ouvert la voie avec Marcher, une expérience de soi dans le monde : essai sur la marche écoformatrice. Cette année, c'est Gaston Pineau qui essaie d'exprimer les acquis de ses six voyages, dont cinq à vélo, qui ont ponctué ses dix premières années de retraites. Pascal Galvani, codirecteur avec Dominique Cottereau de la collection Écologie et formation (chez L'Hamattan), en écrit la postface : Vivre en trans-formation permanente (p. 183-192). Après avoir explicité la tresse des multiples dimensions de la formation humaine, l'ouverture émancipatrice de la formation existentielle dans la vie quotidienne, la sympathie des éléments et les métamorphoses du vivant, il termine en synthétisant les éléments pour une exploration du "Ça crée le Soi », aïku final de Gaston, synthétisant l'apport de cette première expérience de la retraite comme voyage ultime et crucial d'autoformation avec le monde.

9 La mondialisation bouleverse les équilibres écologiques, mais ouvre aussi les horizons interculturels des formations/transformations possible. Le récent livre de Pascal Galvani, Autoformation et connaissance de soi (2019), explicite magistralement les assises théoriques, phénoménologiques et herméneutiques, qui animent le mouvement d'écoformation. Ce dernier est l'un des trois mouvements qui accompagnent l'émergence d'un paradigme systémique et dialectique, d'une formation en deux temps. - expérientiels/formels - et trois mouvements - entre soi, les autres et les choses. "L'exploration de l'autoécosocioformation ouvre la compréhension de soi à la prise de conscience des interactions globales et planétaires qui assurent notre survie. " (Présentation du livre)

Pour prendre au sérieux cette métamorphose des théories et des pratiques éducatives qu'impose l'ouverture des apprentissages tout au long et dans tous les secteurs de la 
vie, Hartmut Rosa l'alimente triplement avec son ouvrage de 2018 : Résonance. Une sociologie de la relation au monde. D'abord avec sa définition de la formation :

La formation, au sens où l'entend la théorie de la résonance, ne vise pas plus à une formation du monde qu'à une formation de soi, mais bien à une formation de la relation au monde. Son enjeu n'est pas le perfectionnement individualiste et atomiste de soi, non plus qu'une maîtrise désengagée du monde, mais l'ouverture et l'instauration d'axes de résonance. (Rosa, 2018, p. 276)

Ensuite, avec cette ouverture active à des axes de résonance, il propulse enfin le concept de résonance, à travers et au-delà des raisonnements arraisonnant. Il remet la tête sur les épaules d'un corps sensible situé socialement et spatio-temporellement entre ciel et terre.

La résonance est l'affleurement (momentané) d'une connexion à une source d'évolutions fortes au sein d'un monde principalement muet et souvent répulsif... L'effet produit par ces expériences est donc moins de confirmation... qu'une transformation : le contact implique une fluidification de la relation au monde, de sorte que le moi et le monde ressortent toujours transformés de leur rencontre. (p. 212-213)

En faisant de ces expériences de résonance l'envers de l'aliénation (p. 215), l'auteur privilégie la formation expérientielle avant la formation formelle, l'objectif de cette dernière étant de mettre en forme et en sens les expériences sensibles.

Enfin, la partie II (p. 223-353) explore la formation de relations à la complexité d'un monde selon trois sphères - objective, subjective et sociale - et trois axes de résonance : axes horizontaux (famille, amitié, politique); axes diagonaux (relations aux objets, travail, école, sport et consommation); axes verticaux (promesses de la religion, voix de la nature, force de l'art, manteau de l'histoire.)

L'ouvrage est construit en quasi-exclusivité avec des auteurs non-francophones. Mais cette sociologie de la relation au monde apporte de l'eau au petit ruisseau d'une philosophie de la résonance et du retentissement qui, depuis Gaston Bachelard, alimente de façon souterraine la voie buissonnière émergente de l'écoformation au cours de la vie. Le dernier ouvrage de Bernard Honoré - Histoire de vie et formation de la personne (2019) - en est actuellement l'expression la plus vive. Ça bourgeonne !

\section{BIBLIOGRAPHIE}

Bézille, H., Froissart, T.et Legendre F. (2019) Qu'apprendre de la formation des artistes de cirque? L'expérience Fratellini : une histoire d'écoformation professionnelle en devenir. Paris : l'Harmattan, collection « Écologie et formation »

Blondeau, N., Boy, V. et Potolia, A. (coord.) (2019). L'école sans murs. Une école de la reliance. Paris : l'Harmattan, collection « Écologie et formation »

Cottereau Dominique (coord.) (2017). Dehors. Ces milieux qui nous trans-forment. Récits écobiographiques nés d'ateliers d'écritures. Paris : l'Harmattan, collection « Écologie et formation » Galvani, P. (2019). Autoformation et connaissance de soi. Lyon : Chronique Sociale 
Honoré, B. (2019). Histoire de vie et formation de la personne. Paris : L'Harmattan, collection « Histoire de vie et formation »

Moneyron, A. (2018). Agroécologie. Quelle écoformation ? Paris : l'Harmattan, collection « Écologie et formation »

Pineau, G. (2019). Voyages, retraite et autoformation mondialogante. Paris : l'Harmattan, collection "Écologie et formation"

Pineau, G. (2018). Apprendre l'ère planétaire à partir de lieux singuliers. Tour de l'hémisphère nord inspiré par le sud (25 mars - 15 mai 2018). Montréal : Éditions Maletto

Rosa, H. (2018). Résonance. Une sociologie de la relation au monde. Paris : La Découverte

\section{AUTEUR}

\section{GASTON PINEAU}

Chercheur émérite au Centr'ERE 